\title{
A REVIEW ON PURIFICATION OF FLUE GASES BY USING BOTH VENTURI SCRUBBER AND CYCLONE SEPARATOR
}

\author{
Dr. Suresh Kumar. R, \\ Head of the Department, \\ Sri Eshwar College of Engineering, Coimbatore 641202, India \\ Raakesh.M, Shanketh.S, Thaaranidevi.C. K, Udhayakumar.S \\ Department of Mechanical Engineering, \\ Sri Eshwar College of Engineering, Coimbatore 641202, India
}

\begin{abstract}
The venturi scrubber and cyclone separator are the devices, which is mainly used to control air pollution in the environment. These devices are very useful in purifying the contaminated air and the dust particles. In this literature it is found that the performance of venturi scrubber (i.e., collection efficiency) is mainly influenced by the injection of droplets at throat section, pressure drop, inlet velocity of the gas and droplet distribution. The performance of the cyclone separator is affected by dimensions of inlet and outlet ducts, inlet flow velocity and particle size distribution in feed. By the uniform distribution of droplet in venturi scrubber its performance will be improved. The collection efficiency of the cyclone separator is completely related with the design parameter and operation parameters. To improve the collection efficiency of the contaminant air and dust particle venturi scrubber and cyclone separator are combined in a single setup. This review shows the experimental study of venturi scrubber and cyclone separator.
\end{abstract}

Keywords: Venturi Scrubber, Cyclone Separator, Contaminated Air, Dust Particles, Collection Efficiency.

\section{INTRODUCTION}

Now a days, the pollution norms are getting more strictness to save the environment. Due to these norms industry are adopting new pollution control techniques. The venturi scrubber and cyclone separator are the best solution to control the pollution viz, Casting Units, Cement Plants, Sugar Factory and Nuclear Power Plants. These pollutants causes health issues to the human beings and even very hazardous to animals and plants. To meet out the necessity of the industry it is the effective method to remove the pollutants.

Venturi scrubber is a device used to control the gaseous emission and particulate matter which is suspended in a gas stream. A venturi is composed of three sections, viz convergent section, throat section and divergent section. In convergent section, the contaminated gas from the exhaust of industry enter with the maximum velocity. In throat section, the liquid is introduced in spray form by the external force. The liquid and gas comes in contact each other. This result in production of large number of droplets by atomization of contaminated gas at high flow rate. In divergent section, gas gets decelerated.

Cyclone separator is a device used to separate the dust particles by creating centrifugal force inside the system. It consists of two parts: upper cylindrical part, lower conical part. It works by forcing the particulates to flow into cylindrical space, so that particles will deposit in the conical surface of the cyclone separator and the remaining air will be led out. This is also used to remove the droplets of liquid from the gaseous stream. Particle with critical size will give great efficiency.

\section{LITERATURE REVIEW}

\section{A. VENTURI SCRUBBER:}

Siddhi Jadhav, et al.,[1] stated that assortment effectiveness, pressure drop across venturi scrubber and assortment productivity of oneself preparing venturi scrubber were broke down by utilizing exploratory technique. The venturi scrubber was one of the effective gadgets to expel the particulate issued from a gas stream. In self-preparing venturi scrubber injection of scouring fluid happened because of contrast in hydrostatic weight of fluid and static weight of gas at the throat segment. The venturi scrubber encased dust molecule and little water beads streaming into it. When water head increased over the venturi scrubber weight drop across venturi scrubber reduced. Assortment productivity of self-preparing venturi scrubber incremented with increment in water head over the venturi scrubber.

Safdar, et al., [2] proposed CFD simulation on the study abatement process of Sulphur dioxide through chemical 


\section{International Journal of Engineering Applied Sciences and Technology, 2020 Vol. 4, Issue 11, ISSN No. 2455-2143, Pages 167-178 \\ Published Online March 2020 in IJEAST (http://www.ijeast.com)}

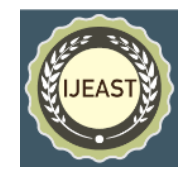

reaction with water holding inside a venturi scrubber. Based on the Environmental Protection Agency (EPA) standards different industries have employed various means of measures to control the hazardous flue gases emitted from the coal utilized for their process. Out of the different scrubbing technologies available in the industrial arena venturi scrubber was found to be having the ability to remove toxic gases and particulates simultaneously. In this study, computational fluid dynamics (CFD) techniques was used to understand the behaviour of gases inside scrubbing technologies. Mass concentration of sulphurous acid, Sulphur dioxide and water mass content distribution inside venturi scrubber were few parameters that are considered for the analysis. The investigation was also concentrated towards the removal of sulphur dioxide. The study concluded on the note that the water mass content distribution was greatly influenced by Sulphur dioxide mass flow rate. However, an increase in sulphur dioxide resulted in decrease of desulfurization efficiency.

In a venturi scrubber, $\mathrm{SO}_{2}$ mass flow rate significantly regulates the distribution of water mass concentration which allows the reaction to start either only in diffuser section or throughout a venturi scrubber. Initiation of reaction in different sections of the venturi scrubber is due to the presence of lower $\mathrm{SO}_{2}$ concentration and presence of higher concentration results in initiation of reaction in the diffuser section of the venturi. Another factor, $\mathrm{L} / \mathrm{G}$ ratio also plays a vital role in the removal of $\mathrm{SO}_{2}$. It is noteworthy to state that the removal efficiency increases with an increase in $\mathrm{L} / \mathrm{G}$ ratio.

AdiSurjosatyo, et al., [3] showed the correlation between optional warm splitting and venturi scrubber so as to decrease tar focus. In any case, one of the significant difficulties in utilizing syngas as an electric force was a tar issued as a gas that can cause fouling in pipes. The study was performed in $10 \mathrm{~kW}$ rice husk biomass gasifier. The greatest tar decrease efficiencies of $89 \%$ in venturi scrubber and optional warm breaking of $74 \%$ was recorded. The ideal effectiveness of the venturi scrubber was recorded at $89 \%$ with 3.78 LPM. The presence of the water in the venturi restrained the expansion of the steam rate past 3.78 LPM. The optimum level was attained at $74 \%$ as the ER builds the tar assortment productivity was likewise increments.

ManishaBala, et al., [4] built a reasonable model to expel the $\mathrm{HCl}$ from off gases. The proposed model utilized a self-priming venturi scrubber. The parameters considered are throat gas speed, fluid level in the external chamber and channel grouping of $\mathrm{HCl}$. The study involved typical water as the cleaning fluid. The parameters are assigned with the ranges viz throat gas speed $(36-72 \mathrm{~m} / \mathrm{s})$, fluid level in external chamber $(0.40-0.77 \mathrm{~m})$ and channel grouping of $\mathrm{HCl}(100-500$ ppm). The study also included the utilization of $\mathrm{NaOH}$ arrangement as a cleaning fluid in submerged and non- submerged conditions. The maximum productivity of $\mathrm{HCl}$ was achieved at $87.83 \%$ when ordinary water was used as the cleaning liquid. The usage of $\mathrm{NaOH}$ showed the maximum limit at $92.54 \%$ with $\mathrm{NaOH}$ concentration maintained within $0.005 \%$. The above results were achieved when the parameters are assigned with the level of throat gas speed of $60 \mathrm{~m} / \mathrm{s}$ and fluid degree of $0.77 \mathrm{~m}$ in submerged condition. It was likewise finished up from the outcomes that the $\mathrm{HCl}$ expulsion proficiency was higher for higher gas stream rate, higher fluid level in external chamber. What's more, it likewise increments with the expansion in bay $\mathrm{HCl}$ focus. Besides, exact models were created to anticipate the $\mathrm{HCl}$ expulsion effectiveness for submerged and nonsubmerged conditions with ordinary water and $\mathrm{NaOH}$ arrangements as cleaning fluid. Observational models strayed extremely less from the trial results. The benefits of self-preparing venturi scrubber over the multistage double stream sifter plate wet scrubber system done by Kurrela et al. This was that, framework will keep on working in uncommon conditions like force disappointment circumstance and so on. The present framework was minimized and prerequisite of room was more if there should be an occurrence of multi-organize double stream strainer plate wet scrubber. So present examination recommends oneself preparing venturi scrubber with antacid as a cleaning fluid can be a decent alternative to expel the lethal $\mathrm{HCl}$ gas from the air to spare the human life.

RushikeshGhale, et al., [5] paper talked about wet scrubbers require less support and create less emanating than regular dry scrubbers while expelling corrosive gases, for example, hydrogen chloride, hydrogen fluoride and nitric corrosive from pickle line rage exhaust streams. Contamination control necessities had become so exacting now, that numerous producers were understanding that the best gushing was no emanating, and that the 'zero release' idea has numerous attractions. So as to have zero release, the set-up methods for doing things must be re-evaluated and improved in view of this item. Generally, rage scrubbers for pickle lines have utilized huge volumes of water. This paper was about wet scrubbers, which moderate measures of water, and produce a generally solid corrosive, that can be came back to the pickling procedure, rather than being killed. The majority of the discourse will allude to hydrochloric corrosive $(\mathrm{HCl})$ scrubbers, since this corrosive was the most generally utilized pickling corrosive, and $\mathrm{HCl}$ was focused as one of the controlled mixes of Title III of the United States' 1990 Clean Air Act. Scrubbers were just a piece of the arrangement; improved smoke control frameworks which limit the fumes streams and contaminant loadings were similarly as significant - the 'fumelesspickler'. Consequently, we had contemplated working of the wet scrubber to limit the HCL discharge to the air and to accomplish ideal productivity we included chevron cutting edges with water showers after 


\section{International Journal of Engineering Applied Sciences and Technology, 2020 \\ Vol. 4, Issue 11, ISSN No. 2455-2143, Pages 167-178 \\ Published Online March 2020 in IJEAST (http://www.ijeast.com)}

the pressing material by the utilization of which effective measure of HCL in the fumes air was expelled.

ManishaBal, et al., [6] proposed a regulating venting framework (FCVS). The proposed model was a basic innovation in atomic force plants for the evacuation of iodine. The main sourcing fluid for throat gas speed of $18 \mathrm{~m} / \mathrm{s}$ and fluid stream pace of $0.033 \mathrm{~kg} / \mathrm{s}$ was determined by effectiveness of iodine as $82.32 \%$ at $3 \times 10^{-3} \mathrm{k} \mathrm{mol} / \mathrm{m}^{3}$ of $\mathrm{KI}$ arrangement. The expulsion effectiveness of iodine was anticipated by a semi-exact model which cleans and utilises the effect on the scrubber. From the exploratory outcomes, the most extreme expulsion productivity of the iodine was acquired as $70.13 \%$ with typical water as a cleaning fluid at the throat gas speed of $18 \mathrm{~m} / \mathrm{s}$. After expanding the centralization of $\mathrm{KI}$ in the scouring fluid, iodine evacuation effectiveness increased because of expanded dissolvability of iodine in $\mathrm{KI}$ arrangement. It was additionally inferred that iodine evacuation effectiveness was higher for higher gas stream rate, higher fluid stream rate and higher gulf centralization of iodine. A relationship had been enveloped for anticipating the expulsion productivity of iodine with water as a scouring fluid and test results are in great concurrence with this connection.

Avinashmoharana, et al., [7] proposed an experiment on venturi scrubber working in self-preparing mode. The proposal in based on the venturi scrubber with three stage stream collaboration. The model consolidated the impact of bead crash and expanded force source in the high fluid focus. The anticipated neighbourhood fluid stacking, pressure drop and the assortment effectiveness were seen as in great match with the referred to exploratory qualities. The study relates the parametric enquiry for various throat gas speed, fluid infusion speed and changing opening distance across the pertinence to make self-preparing mode in the scrubber. The fluid transition profiles and the performance parameter are contrasted with study the impact of various working and geometric parameters on the scrubber's general effectiveness. Then again, the bead size was seen as a solid capacity of gas speed where enormous number of littler beads helped with accomplishing higher assortment productivity. The pivotal weight profiles followed a monotonic pattern of expanded weight drop with the expansion in the fluid burden to the scrubber. The performance parameter fills in as a novel explanatory instrument to relate the hydrodynamic conduct happening in the throat segment with the general assortment productivity of the scrubber. It is discovered that with increment in the scrubber effectiveness there was an expansion in the estimation of performance parameter.

The venturi scrubbers gave profoundly efficient expulsion of particles from dust-loaded gases. Most computational models used to anticipate the scattering of fluid [8-14] in scrubbers utilize the Boll connection to figure the mean bead size. Trial estimations of bead size circulation and fluid scattering were made under different conditions. The fluid dynamic was reproduced with ANSYS Fluent 14.0 programming, utilizing a discrete stage direction model. The re-enactments were performed with bead size disseminations estimated tentatively and anticipated utilizing parameters from writing. The outcomes for the fluid scatterings were in concurrence with the trial information and demonstrated a solid reliance of the fluid dissemination in the scrubber on the parameter esteems utilized in the bead size dispersion model. The computational model had the option to sufficiently foresee the profiles of weight and fluid scattering inside the venturi scrubber, and great understanding was gotten with the test results. The bead size circulation had a significant influence on fluid scattering close to the infusion plane. This influence was uncovered by the different fluid scattering profiles acquired, contrasting the infusion of beads considering Rosin-Rammler size appropriations got tentatively and utilizing writing parameter esteems. The movement of the beads to planes increasingly far off from the infusion plane was related with a uniform appropriation of fluid in the throat area, and the difference between the fluid scatterings got utilizing the test and hypothetical Rosin-Rammler conveyances was diminished.

Hassan Ali, et al., [15] did an experiment on radiating wet scrubber designwith computational investigation of stream. The experimentalist makes a productivity collection of dust $>10 \mathrm{~mm}$ in breadth to $99.9 \%$ bargained effectiveness at low loads and high loads. Desulphurization towers were used to check the issues in pipe gas. The study enquired the different processing plant conditions with scrubber presentation. The revealed issues were found with the help of diverse scrubber stream parameters and measurements. To make the fulfilment of complete plan upgrades the urgent of elite made with CFD discoveries and an improved comprehension of the scouring fluid stream design. Lab scale tests were done to estimate the prescient capacity of ANSYS Fluent for 1 and 2 stage stream. The CFD recreations had the option to anticipate the large scale stream designs yet the single stage re-enactments under expected the air speeds close to the mass of the SSM with all the disturbance models utilized. The expectations utilized the RSM gave the nearest coordinate with exploratory outcome. The 2 stage re-enactments under anticipated the weight drop over the SSM by roughly $10 \%$. The anticipated speeds of beads through the demister vanes of the SSM were steady with the drop speeds controlled by fast photography.

ManishaBal, et al., [16] derived a study with venturi scrubber and work channel to make an applicable framework of separated control venting framework (FCVS).To make the full utilization and current 


\section{International Journal of Engineering Applied Sciences and Technology, 2020 \\ Vol. 4, Issue 11, ISSN No. 2455-2143, Pages 167-178 \\ Published Online March 2020 in IJEAST (http://www.ijeast.com)}

execution of venturi FCVS method must be surveyed with addition to all. In addition to the above process the improvement of venturi scrubber plan was done by hydrodynamics. In the recent years it is noticed that computational fluid dynamics (CFD) was the key feature in utilizing the hydrodynamic conduct of venturi scrubber. Under the various stream conditions in the venturi scrubber the weight drop profile anticipated with gambit 2.4.6 and Ansys familiar 15. The re-enactment was utilized with the volume of the liquid (VOF) and Reynolds Renormalization Group (RNG) k- $\varepsilon$ disturbance model with higher efficiency. The least weight drop of 373.51 was made with the throat gas speed of $24 \mathrm{~m} / \mathrm{s}$ and fluid stream phase of $0.016 \mathrm{~kg} / \mathrm{s}$ and the nominal weight drop 2064.34 was made with the throat gas speed of $60 \mathrm{~m} / \mathrm{s}$ and fluid stream phase of $0.033 \mathrm{~kg} / \mathrm{s}$. The main purpose of building a venturi scrubber was to be utilized as an air contamination controlling device and dust cleaning device. The augmented gas speed was created in little beads to clean fluid water at the throat segment. The pressure drop inside the venturi scrubber was made with the property of hydrodynamics in numerical model analysis. To examine and predict the actions of the hydrodynamics familiar programming was utilized. The parameters like fluid mass stream rate, throat gas speed and fluid to gas proportion were changing to examine hydrodynamic property.

AvinashMoharana et al., [17] presented a study on three phase flow development in the venturi scrubber with computational model simulation. The arrangements of droplets and aerosols were made with Euler-Lagrangian in acomputational model. In the high liquid concentration region makes the increased momentum and mainly incorporates the effect of collision in droplets. The droplet size distribution was included in prediction for droplet inertial effects in multi-jet. Within all the sited experimented values the required details were calculated with local liquid loading, pressure drop and the collection efficiency. To make the characteristics parameter multi-jet at the throat region of the venturi scrubber the unique dimensionless number called performance parameter was made with jet penetration and droplet mean diameter. To make a proper assessment for the performance parameter an analytic tool is obtained as high efficiency in scrubber from the high values of performance parameter. The variable injection diameter and multi-stage injection was advanced by lower gas velocities and lower operating height. The jet penetration and the droplet size distribution was characterised on the basis of the local liquid loading ratios and the droplet SMD in the throat section of the venturi scrubber and was quantified by evaluating the performance parameter for the prevailing condition. The liquid flux profiles and the performance parameter were compared to study the effect of different operating and geometric parameters on the scrubber's overall efficiency. From the results of analysis, it found that, higher jet penetration obtained for high liquid loads (and/or) higher nozzle diameter helped in increasing the collection efficiency. On the other side, the droplet size was found to be a strong function of gas velocity where large number of smaller droplets assisted in achieving higher collection efficiency. The axial pressure profiles followed a monotonic trend of increased pressure drop with the increase in the liquid load to the scrubber. It is found that with increase in the scrubber efficiency there was an increase in the value of Performance Parameter.

SiriwatUnyaphan, et al., [18] made an experiment with regenerative unit and $2 \mathrm{~L}$ of canola oil in the lab-scale test for the better utilization of the venturi scrubber in these process. This lab-scale test was made for the issue in fouling and forestall stopping in the control of the tar fixation to adequate level in the biomass gasification advancement. To make the tar evacuation execution for long haul a retentive recovery was projected towards the venturi scrubber with minimal effort. The tar evacuation was contrasted $18 \%$ with foaming scrubber in the venturi scrubber with syngas micro bubbles. The maintaining of expulsion proficiency in tar could be kept up somewhere in the range of $90 \%$ and $96 \%$ along 10 hours explore by retentive recovery. Additionally, each time the canola oil was recovered, the tar expulsion execution was recouped to over $90 \%$ which was nearly equivalent to the crisp canola oil performed effectiveness. The tar evacuation in the venturi scrubber was $98 \%$ which makes much higher than that of percolating scrubber by $18 \%$. The higher oil throat speed delivered micro bubbles which viably improved the tar evacuation execution because of the expansion of the reaching zone and the maintenance time. At that point, there was oil recovery unit in the venturi scrubber with better utilizing of canola oil in the activity without making the replacements in the permeable oil.

Zhenhui Luan, et al., [19] created an artificially broke down for assortment of tar evacuation strategies to the flat shower venturi scrubber from the gas. The created model was made by programming CATIA with the cylinder shape and calculated the speed shapes, pressure forms and fierce active vitality by choosing the ANSYS-FLUENT with numerical re-enactment of the cylinder model. The outcome made up the cylinder model is showed such focal points as large speed throb, enormous weight misfortune, huge fierce active vitality, etc. Thus, it had a high proficiency of tar evacuation, and had an application prospect in building venture. The creators set up a cylinder model of venturi tube with flat shower makes an auxiliary parameters that tends to recreate the highlights of stream field, pressure and divider shear. With the help of cylinder model contrasting speed, weight and divider shear of those downstream shower of level splash had been gained in greater speed throb, prominent pressure and decoking purging proficiency was also increased. 


\section{International Journal of Engineering Applied Sciences and Technology, 2020 \\ Vol. 4, Issue 11, ISSN No. 2455-2143, Pages 167-178 \\ Published Online March 2020 in IJEAST (http://www.ijeast.com)}

Futang Xing, et al., [20] experimented a study that describes the pollution from the modern pipe gas sanitization which was extremely suitable with venturi scrubber as effective gas cleaning gadgets. The residue evacuation productivity was considered with the gas entering at specific velocity inside the scrubber. The resulting consistency of speed conveyance was checked numerically. An enhanced plan for an overwhelming hill venturi was proposed. The profiles of both the hill and cylinder are illustrative. Contrasted and the traditional structure, the bigness territory be-comes directly customizable, which has benefits for improving the productivity and diminishing the commotion of the venturi tube. The CFD recreation confirmed that the upgraded structure displayed a progressively uniform gas speed appropriation.

The experiment describes that venturi scrubber gets absorption efficiency with the performance of iodide vapour, depends on both experimental and theoretical calculation [21-27]. The calculations based on the experimental results indicated flow rate, gas flow rate and temperature which was very likely to be noticed as absorption efficiency. The experimental values cannot meet up the requirements of insensitive to iodide inlet concentration. By making the variation in gas and liquid contacting time, the gas flow rate on the absorption efficiency was improved in main cases. The absorption efficiency gets reduced due to low flow rate in the solution. Moreover, temperature was found to be insignificant when the absorption efficiency gets improved by the constant gas flow rate in the venturi. The result becomes a satisfied one, when the solution rate increases with lower in flow rate. The result with vice versa, decreased in solution rate with higher in gas flow rate leads to neglect gas and liquid phase in transverse exchange. The proposed mathematical model gave favourable predictions in the whole range of experimental conditions. Furthermore, the predicted accuracy was more satisfactory with high aqueous solution flow rate. The most optimal size can be determined from mathematical calculation to ensure the absorption performance and reduce pressure loss at the same time when the removal efficiency was required.

N. P. Gulhane, et al., [28] presented research on 3 dimensional displaying to break down weight and speed of liquid at the throat of self-preparing venturi scrubber. That the separated vented control framework (FVCS) containing venturi scrubber was being introduced so as to forestall the particulate issue and vaporous contamination entering the earth because of extreme mishaps in atomic force plant. Then the experiment is taken pace with programming "ANSYS WORKBENCH" which would be more suitable to do all the instrumental works. From the force preservation standard, it is discovered that the utilization of $\mathrm{K}-\mathrm{C}$ choppiness model the speed field of gas stream was obtained. Gas and fluid collaborated with one another in the throat area of venturi scrubber, consequently the weight, speed of liquid at the throat and weight drop across venturi scrubber were the key elements to decipher the exhibition of venturi scrubber. The 3 dimensional model of venturi scrubber was considered having throat length of $0.038 \mathrm{~m}$ and width $0.019 \mathrm{~m}$. The outcomes from present examination and recreation were useful to improve the venturi structure. CFD investigation of self-preparing venturi scrubber was accomplished for the investigation of hydrodynamics of gas-fluid inside the venturi scrubber by utilizing EulerEuler approach. The weight at throat assisted with understanding volumetric fluid to gas proportion inside venturi scrubber. This work was useful to improve the venturi structure for good execution.

\section{i.FINDINGS IN VENTURI SCRUBBER:}

1. Cleaning of flue gas emitted from coal burning can be done by venturi scrubber as it have the ability to remove particulates and toxic gases simultaneously.

2. Self-priming venturi scrubber with antacid as a cleaning fluid can be a decent alternative to expel the HCL gas from the air to save the human life.

3. The computational models used to anticipate the scattering of fluid in scrubbers utilize the Boll connection to find the mean bead size.

4. Hydrodynamics was a significant way that should be examined to improve the plan of the venturi scrubber.

5. The scrubber with inlet velocity makeskey element which affecting its residue evacuation productivity.

6. The gas flow rate on the absorption efficiency was mainly influenced by the variation of gas and liquid contacting time.

\section{B. CYCLONE SEPARATOR:}

Jihe Chen et al., [29] broadly utilized detachment gadget to improve its partition efficiency. The study involved two-arrange tornado separator and demonstrated by the Reynolds stress model. The distance across of the 2ndorganize twister (D), the effects of five 1st-arrange violent wind breadths $(\mathrm{Du})$ on the demonstration of the 2-organize tornado are recreated. The outcomes exhibited that Du has significant effects on the interior weight field, flow field and vortex centre of the twoarrange violent wind. Contrasted and the 1-arrange violent wind separator, the division efficiency of the 2organize twister separator was significantly improved. When $\mathrm{Du}=6 \mathrm{D}$, the partition efficiency was upgraded by $15.5 \%$ contrasted and that of the 1 -arrange violent wind. Moreover, the 2-arrange twister separator can effectively diminish the Euler number. The outer violent wind distance across $(\mathrm{Du})$ has a significant effect on the weight field, flow field, and vortex centre of the 2arrange twister, and the effect on the 1-organize tornado was significantly more than that of the 2-arrange typhoon. The 2-organize violent wind separator has 


\section{International Journal of Engineering Applied Sciences and Technology, 2020 \\ Vol. 4, Issue 11, ISSN No. 2455-2143, Pages 167-178 \\ Published Online March 2020 in IJEAST (http://www.ijeast.com)}

higher partition efficiency than the 1-arrange typhoon separator.

M.N. Nwinbo [30] made an experiment to determine the control in wood handling shops with a testing of a Saw residue Cyclone separator. The experiment creates a cyclone separator with $14.7 \mathrm{~m} / \mathrm{s}$ by the usage of Lapple model. Saw residue of various molecule sizes was utilized as a test to assess the typhoon execution. The structured twister was contrasted and a standard high effectiveness tornado, and it found hypothetically to work in better productivity under indistinguishable working conditions. Execution assessment and computational examination displayed the particulate size, static weight drop, delta particulate speed, tornado measurements and particulate focus in air were the fundamental factors in assessing the violent wind assortment execution. The twister particulate assortment effectiveness incremented with expanding particulate size. The experiment shows huge breadth tornados were best for expelling enormous particulates from a huge particulates-loaded gas stream. Tornado separator was mechanical framework that control particulate discharges by utilization of divergent partition process. Static weight drop was the most significant factor in assessing the exhibition of this contamination control gadget. The violent wind particulates assortment productivity incremented with expanding particulate size. By this way more breadth violent winds were best for expelling more particulates from a more particulates-loaded gas stream.

Saputro H et al., [31] investigated the performance of Refuse Derived Fuel (RDF 5) by palletisation process. The RDF 5 of palm starch squander was utilized as the biomass fuel in the gasifier. In any case, the Syngas quality by utilizing the little scale gasifier in the research center of Mechanical Engineering Education indicated the contamination. It was because of different results of gasification procedure, for example, tars, particulates (Roast Debris and Residue) and water substance of gas. Accordingly the point of this investigation was to locate the appropriate of the tornado separator in the gasification contraption that can isolate the syngas and particulates. So as to improve the nature of Syngas from the particulates adulteration in gasification process of palm starch squander, countercone could be applied to the violent wind separator. The after effects of this regeneration show that by including the counter-cone in the fierce wind separator increasing the quantity of particulates caught and the appropriation yield of molecule measurement. The extra of countercone in the violent wind separator was acceptable approach to diminish the disturbance toward the finish of the vortex the geometry of the twister separator type $\mathrm{C}$ show the best effectiveness contrasted and geometry $\mathrm{A}$ and geometry B. Consequently, the geometry type C is reasonable to the trait of gasification procedure of palm starch squander.
The execution of violent wind was influenced by two imperative parameters [32-36], those were specifically assortment proficiency and weight drop. The two parameters were firmly affected by the geometry of the gadget. The geometric parameters that influence its presentation were for the most part stature of funnel shaped area and chamber, barrel breadth, vortex distance across, vortex discoverer length, cone plunge width and so on. Rahul Panchal, et al., states that predominantly centres around the geometric parameters of a twister and dissecting its impact on the assortment productivity. Rahul Panchal, et al., used three distinctive size tornado dust authority models. The impact of violent wind size on execution parameters, for example, pressure drop, cut off width, and assortment proficiency was inspected. The CFD examination of three violent wind dust gatherers was performed. The impact of violent wind size was numerically researched to contemplate the presentation parameters and hydrodynamic qualities of the stream. The created CFD model spoke to stream directions of liquid and the vortex. The re-enactment results uncovered that with expanding the violent wind size the typhoon weight drop and cut off breadth will increases.

ForamMaheshwari, et al., [37] to exhibit the twister separator can be estimated the assortment effectiveness and weight drop. Parameters like Inlet Flow speed, the molecule size appropriation in feed, measurements of gulf and outlet conduits and violent wind influences the exhibition of tornado altogether. Different Mathematical models utilized for figuring cut off width of separator, stream rate, target effectiveness and no. of vortex inside the typhoon to structure and concentrate to check the exhibition of existing violent wind separator. Here, in this examination the effectiveness accomplished with Lapple model in total $86.47 \%$. Impact of assortment productivity and weight drop on the typhoon execution was considered utilizing the created Lapple Model. Diminishing the littler molecule size gives less quantities of proficiency of detachment particles. It was reasoned that for littler size molecule from the range $60 \mu \mathrm{m}-10 \mu \mathrm{m}$ less assortment proficiency accomplished. The model expectation was inacceptable concurrence with Lapple model with study hypothetical writing study with other writer's discoveries which builds the creatibility of the strategy. For accomplishing great outcomes between hypothetical presumption and trial information the Lapple hypothesis parameter had great strategy to follow. In the Leith-Litch hypothesis information was not coordinate with the hypothetical fragmentary effectiveness bends. So, Leith-Licht and Barth hypotheses worked better for staimand higheffectiveness, yet when measurements changes because of gas stream designs in the twister separator, and hypothetical suppositions that function admirably for one structure may not be substantial for another. The 


\section{International Journal of Engineering Applied Sciences and Technology, 2020 \\ Vol. 4, Issue 11, ISSN No. 2455-2143, Pages 167-178 \\ Published Online March 2020 in IJEAST (http://www.ijeast.com)}

speculations should be changed for an alternate scope of violent wind structures and working conditions.

Ismail khan, et al., [38] said that rice was the critical sustenance for most of the people in Asian country, it was the greatest eaten up calorie source among the sustenance grains. Rice plant was one of the most prepared and greatest agro taking care of organizations in our country. The methodologies used in adventures for changing over paddy to the kind of rice incorporate cleaning, hulling, lighting up, etc. The rough material which will be gotten by the plant contains even more generous build-up partials, fine build-up particles, minimal estimated stones and various other minimal waste particles. Milling was the place where paddy was changed into rice which was proper for human use, during this system significant amount of build-up particles was left to the atmosphere which was particularly dangerous to the people respiratory construction and as a result of the respiratory issue an huge number of them had lost their life, the most unsafe part was that, the people who was working in rice processing plant will be influenced first. Recognizing these issues we are needing to use storm separator with channel unit and blower to control the build-up particles which is liberated in the various spots of the rice processing plant during the methodology. The present Project was done by four people. A libbed tornado separator had been made for grouping and detaching bui;ld-up particles before allowing it to nature. The made twister separator has been adequately used as late organized channel unit for controlling buildup in Rice Mills. This late arranged tropical storm separator can be combined successfully for cleaning.

AbhijeetGayakwad, et al., [39] predicted the cyclone performance in terms of velocity and pressure variation, a large number of computational study was conducted due to its much application in the industrial area. They stated many industries were used the cyclone dust separators without any modifications for more than a century. The gas solid cyclone separator was widely used in industries. The simulation was carried out using computational fluid dynamics (CFD) for gas particle flow with cyclone separator in one of the approaches. Most of the attention was focused on improving the cyclone performance parameters. Recently most of the studies focused on the geometric effect on the cyclone performance. In this experiment explains that the geometric effect on cyclone separator was studied with the creation of symmetrical tangential inlet cyclone separator and compared it with the classical cyclone separator. The results showed that the new geometric modification to the cyclone improves the performance. The collection efficiency for the modified cyclone geometry was more than the standard cyclone design. The results of pressure contour show uniform distribution of pressure throughout the cyclone body when compared to the standard design.
The tornado separator was a kind of mechanical residue authority that was utilized to change channel strong particles from the approaching gas stream [40-47]. The two essential parameters that influenced the exhibition of a twister separator were the assortment productivity and weight drop through the framework. Both assortment proficiency and weight drop were unequivocally affected by the geometry of violent wind separator. The geometric parameters that influence its presentation were basically chamber tallness, cone stature, channel segment, plunge tube stature and so forth. The fundamental point was to expand assortment effectiveness and limit pressure misfortune. This Experiment consider about predominantly centres around varieties of geometric parameters of a typhoon and breaking down its impact on the assortment proficiency. Author states that paper the real violent wind separator model was first approved by utilizing test results and results acquired from the calculations acted in ANSYS CFX. The real model was then adjusted by changing its geometrical parameters like chamber tallness, plunge tube tallness, cone stature and so forth. The CFD investigation of these changed twister separators were performed. Assortment productivity got from the examination was then utilized as a way to choose the last structure of the tornado separator. The model with greatest assortment effectiveness was then chosen. Twister separators were utilized to isolate dust particulates from gas, air or fluid stream. Among all the residue gatherers, violent wind separator had ended up being the most proficient gadget. The general execution of typhoon separator relies to a great extent upon the internal and external vortex framed via air and residue particles separately.

Osezua O. Ibhadode, et al., [48] described that biofuel from any biodegradable development procedure for a nourishment squander bio-digester plant was a blend of a few gases, for example, methane $\left(\mathrm{CH}_{4}\right)$, carbon dioxide $\left(\mathrm{CO}_{2}\right)$, hydrogen sulphide $\left(\mathrm{H}_{2} \mathrm{~S}\right)$, smelling salts $\left(\mathrm{NH}_{3}\right)$ and debasements like water and residue particles. The outcomes were accounted for of a parametric investigation of the procedure of partition of methane, which was the most significant gas in the blend and usable as a biofuel, from particles and $\mathrm{H}_{2} \mathrm{~S}$. A typhoon, which was a customary, financial and straightforward gadget for gas-strong partition which was viewed as dependent on the adjustment of three Texas A\&M violent wind plans (1D2D, 2D2D and 1D3D) by the incorporation of an air delta tube. A parametric measuring was performed of the twister for biogas cleaning, representing the division of hydrogen sulphide $\left(\mathrm{H}_{2} \mathrm{~S}\right)$ and dust particles from the biofuel. The stoichiometric oxidation of $\mathrm{H}_{2} \mathrm{~S}$ to frame basic Sulphur was viewed as a valuable twister plan foundation. The proposed plan incorporates geometric parameters and a few criteria for evaluating the presentation of tornado separators, for example, the Lapple Model for least 


\section{International Journal of Engineering Applied Sciences and Technology, 2020 Vol. 4, Issue 11, ISSN No. 2455-2143, Pages 167-178 \\ Published Online March 2020 in IJEAST (http://www.ijeast.com)}

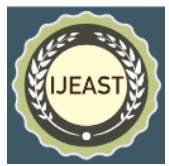

molecule measurement gathered, assortment effectiveness and weight drop. For biogas volumetric stream rates somewhere in the range of 0 and $1 \mathrm{~m} / \mathrm{s}$ and bay stream speeds of $12 \mathrm{~m} / \mathrm{s}, 15 \mathrm{~m} / \mathrm{s}$ and $18 \mathrm{~m} / \mathrm{s}$ for the 1D2D, 2D2D and 1D3D twisters. Separately, it was watched that the 2D2D arrangement was generally monetary as far as estimating (complete tallness and distance across of tornado). The 1D2D design encounters the most minimal weight drop. A structure calculation combined with an easy to use illustrations interface is created on the MATLAB stage, giving an instrument to estimating and structuring reasonable twisters.

Paper displays the plan and execution qualities of a tornado separator utilized in smoked fish preparing [4953]. The use of twister in smoked fish preparing was expected to decrease contaminants, for example, roast, debris, sediment, and a small amount of tar with its polycyclic fragrant hydrocarbon (PAH) compound in the smoked fish. These contaminants possibly decline both item quality and wellbeing of smoked fish. The typhoon utilized was structured by high-effectiveness Stairmand's violent wind to isolate strong particles from the smoke-air stream. A divergent blower of the tornado, which was coordinated with a warmth exchanger, created a constrained smoke-air stream of the backhanded smokehouse. The assortment proficiency of the typhoon was $94.7 \%$, which was higher than the expectation of $93.3 \%$. Visual perception found that tar was caught and clingy at within surface of the violent wind and the residue receptacle. The decrease of these contaminants added to the lessening of the PAH compound, which was demonstrated by low benzo(a)pyrene content (underneath the greatest degree of Turkish nourishment codex for $2.0 \mathrm{~g} / \mathrm{kg}$ ). The absolute weight drop of the typhoon was $204 \mathrm{~Pa}$, which was lower than the anticipated estimation of $332 \mathrm{~Pa}$. The proportion of vitality expended to pressure drop for the violent wind separator was $22.6 \mathrm{MJ} / \mathrm{Pa}$. This effectiveness level has fulfilled the reference bend of execution and safe since the worth surpasses $90 \%$. The molecule size circulation, either the debris particles from stack or debris/dust molecule isolated by the typhoon, had the equivalent littlest size of $0.2 \mathrm{~m}$, which was covering with the smoke particles size. The covering size of particles among debris and smoke showed that division of the debris/dust from smoke-air stream happened from the littlest size to the bigger with the expansion of assortment proficiency. These exhibitions were ensnared to the smoke neatness and made it more advantageous for the fish smoking procedure. The substance of benzo(a) pyrene as an attribute of PAH compound in the smoked fish item was $1.29 \mathrm{~g} / \mathrm{kg}$, which was the low level and safe to expend. Weight drop as a marker of vitality utilization indicated a lower an incentive than that of the expectation and it had a proportion of vitality utilization to the weight drop of the violent wind about $22.6 \mathrm{MJ} / \mathrm{Pa}$. This was to state that the presentation of proficiency, the smoke wellbeing that effect to low benzo(a) pyrene, and the weight drop, it shows that the structured violent wind was ok for useful purposes.

Lin Liu et al., [54] described that impacts of the molecule breadth and molecule infusion position on the gas-strong two-stage stream have significant ramifications in the distracting gulf violent wind separator. The Navier-Stokes condition combined with the Reynolds stress model in Euler facilitate framework was embraced to depict the gas stream and the discrete stage model in Lagrange organize framework was utilized to ascertain the molecule directions. Results showed the partition proficiency changes with the infusion position at the gulf cross-area. It was discovered that the detachment productivity is expanded with the expansion of the molecule breadth. In any case, when the molecule breadth surpasses a basic size, the particles will store on the mass of the funnel shaped chamber, which prompts lower detachment effectiveness. The present reproduction shows that the basic size is $24 \mathrm{~mm}$. The detachment efficiency changes with the infusion position at the delta cross-segment. For a similar size molecule with different starting infusion position, the living arrangement time was different. The more drawn-out living arrangement time, makes the higher plausibility of the molecule divider impact. Consequently, the higher partition efficiency can be delivered. The division efficiency of twister separator was extremely touchy to the changing of molecule breadth. The expanding distance across of molecule prompts expanding division efficiency inside a specific range. In this manner huge breadth violent winds were found best for expelling huge particulates from a huge particulates-loaded gas stream.

The exhibition of assortment effectiveness of round and hollow bay sort twister separator for generally low strong stacking rate conditions [55-59]. Twister separators as a rule work under high strong stacking conditions, however the interest of air contamination control at open air thickly dirtying exercises and use of intensity generators, it is profitable to utilize violent wind separators to control outside air contamination by tornado separators. Evaluation efficiencies and generally speaking assortment efficiencies have been explored from $0.008 \mathrm{~g} / \mathrm{m}^{3}$ to $0.2 \mathrm{~g} / \mathrm{m}^{3}$ strong stacking rates at $5 \mathrm{~m} / \mathrm{s}$ and $10 \mathrm{~m} / \mathrm{s}$ bay speed conditions. Exploratory information were contrasted and two hypothetical forecasts dependent on observational and unthinking connections, created by Smolik and Muschelknautz. To accomplish a superior comprehension of molecule partition productivity, molecule cut size distance across for chosen stacking rate were considered with hypothetical drew nearer by Muschelknautz model. Both test and hypothetical outcomes demonstrated that with the expansion of strong stacking rate and delta speed, the molecule 


\section{International Journal of Engineering Applied Sciences and Technology, 2020 \\ Vol. 4, Issue 11, ISSN No. 2455-2143, Pages 167-178 \\ Published Online March 2020 in IJEAST (http://www.ijeast.com)}

assortment proficiency of twister separator increments. The assortment proficiency under low molecule stacking rates had been examined in present examination for round and hollow bay sort violent wind separator. The examination will be stretched out to research molecule stacking impacts at higher stacking rates and the impacts of broadened container tube for the assortment productivity.

\section{i.FINDINGS IN CYCLONE SEPARATOR:}

1. Static weight drop was the most significant factor in assessing the exhibition of this contamination control in the cyclone separator.

2. Parameters like Inlet Flow speed, the molecule size appropriation in feed, measurements of gulf and outlet conduits and violent wind influences the exhibition of cyclone separator altogether.

3. The twister separator had been adequately used as late organized channel unit for controlling build-up in Rice Mills.

4. A large number of computational study was conducted to predict the performance of cyclone separator in terms of velocity and pressure variation.

5. The collection efficiency of modified cyclone geometry was more efficient than the standard cyclone design.

6. The two parameters that influence the exhibition of a twister separator were assortment productivity and weight drop through the framework.

\section{DUST AND $\mathrm{CO}_{2}$ REMOVAL:}

The effect of electrostatic precipitators (ESPs) commonly applied in air consuming power plants [6063]. Due to the different qualities of build-up removed between oxy-fuel and conventional consuming conditions, a first report has been generated towards evaluation of dust clearing. Fly flotsam and jetsam tests accumulated from a 35 MW oxy-fuel consuming test organize were dissected, and the preliminary of fly trash Resistivity and fly garbage course of action were completed. The difference of the Fly Flotsam and jetsam resistivity under air start was moreover grabbed. The difficulty level evaluation of build-up departure by an electrostatic precipitator under oxy-fuel consuming was less complex than under air start in a specific temperature. Through Research and examination, the arrangement and decision of an ESP under oxy-fuel conditions can insinuate the strategy for standard air consuming. The fly trash resistivity test examination showed that build-up was less complex to be cleared under oxy-fuel start than under air consuming. Under oxy-fuel start, the obvious development speed was found to be sharper and gradually perfect for the ESP.

Gasification test was finished with pilot scale pressurized frothing fluidized bed gasifier [64-65] which was worked with the coal feed cut-off of 500$520 \mathrm{~kW}$. The mix of air, steam, oxygen and $\mathrm{CO}_{2}$ was used as gasification manager with different extents. The working load of gasifier in this examination was some place in the scope of 2.4 and 2.7 bar. The effects of ER, steam/carbon extents and $\mathrm{CO}_{2} /$ carbon extents on syngas structure and cold gas efficiencies were examined. According to the results, using $\mathrm{CO}_{2}$ or $\mathrm{H}_{2} \mathrm{O}$ as gasification products, they may have two inclinations and insults for the action. If $\mathrm{CO}_{2}$ was extended in gasification administrator, reaction was partial unfavourably and $\mathrm{H}_{2}$ things were lessened. The properties and association of the designed gas made by gasification process depend upon various factors of structure and operational parameter (instance, the reactor type, the properties and stream paces of feedstock materials and gasifying administrators, weight and heat in the reactor). The finishes can be drawn from the test outcomes, with the development or decrease of $\mathrm{CO}_{2}$ Flow rate at the bay of the gasifier, unreacted $\mathrm{CO}_{2}$ content in the syngas extended and reduced, by itself. The reaction was also affected by the $\mathrm{CO}_{2}$ content at gasification administrator. If the $\mathrm{CO}_{2}$ content at gasification administrator was extended, reaction balance conditions were changed and results of reaction $\left(\mathrm{H}_{2}\right.$ and $\left.\mathrm{CO}_{2}\right)$ reduced.

\section{i.FINDINGS IN DUST AND $\mathrm{CO}_{2}$ REMOVAL:}

1. Electrostatic precipitators are generated towards evaluation of dust clearing in air consuming power plants,due to the different qualities of build-up removed between oxy-fuel and conventional consuming conditions.

2. Gasification test was finished with pilot scale pressurized fluidized bed gasifier which was worked with the coal feed.

\section{CONCLUSION}

Effect of pressure drop and collection efficiency was studied from this review. The most important factor that influence the pollution control is static pressure drop. While increasing in the particle size, it leads to higher number of separation efficiency. By increasing the water head in venture scrubber the pressure drop will decreases. The larger diameter of cyclone separators are effective for the removal of particulates. The combined performance of the venture scrubber and the cyclone separator leads to high efficiency in collecting of dust particulates and toxic gases reduction.

\section{REFERENCE}

[1] Siddhi Jadhav, KuldipWakade,Nehamagamuni, Ashish Umbarkar. "Investigation of Performance Parameters of Self Priming Venturi Scrubber" Journal of Emerging Technology and Innovation Research, Volume 6.(2019)

[2]Safdar, M.Zubair, N.Iqbal, M.Ali. "CFD simulation of chemical reaction between Sulphur dioxide and water in a venturi scrubber" Separation Science and Technology, 1520-5754.(2019) 


\section{International Journal of Engineering Applied Sciences and Technology, 2020 \\ Vol. 4, Issue 11, ISSN No. 2455-2143, Pages 167-178 \\ Published Online March 2020 in IJEAST (http://www.ijeast.com)}

[3]AdiSurjosatyo, Muhammad BarrylAnggriawan, Andika Akbar Hermawan, HafifDafiqurrohman. "Comparison between secondary thermal cracking methods and venturi scrubber filtering in order to reduce tar in biomass gasification" Elsevier, Science Direct, Energy procedia 158(2019) 749-754.(2019)

[4]ManishaBal,ThamatamTejawani Reddy, B.C.Meikap"Removal of $\mathrm{HCl}$ gas from off gases using self-priming venturi scrubber"

Journal of Hazardous Materials, 364(2109) 406418.(2019)

[5]Mr.RushikeshGhale,Mr.BalajiMundhe,Mr.SaurabhJa iswal,Mr.AdityaYogidas,Prof.SupriyaA.Shilwant.

"Pickling Line Fume Control with Wet Scrubber" International Research Journal of Engineering and Technology, volume 6, Issue 6.(2019)

[6]ManishaBala, ThamatamTejaswini Reddy, B.C.Meikap. "Performance Evaluation of Venturi Scrubber for the Removal of Iodine in Filtered Containment Venting System" Chemical Engineering Research and Design, DOI: https://doi.org/10.1016/j.ched.2018.08.019.(2018)

[7]AvinashMoharana,ParidhiGoel,ArunK.Nayak.

"Performance estimation of a venturi scrubber and its application to self-priming operation in decontaminating aerosol particulates"Nuclear Engineering and Design 320(2017) 165-182.(2017)

[8]VádilaG.Guerra, Ana Elisa Achiles, RodringoBettega. "Influence of Droplet Size Distribution on Liquid Dispersion in a Venturi Scrubber: Experimental Measurements and CFD Simulation" Institute and Engineering Chemical Research.(2017)

[9]Kumar, R. S., Alexis, J., \&Thangarasu, V. S. Optimization of high speed CNC end milling process of BSL 168 Aluminium composite for aeronautical applications. Transactions of the Canadian Society for Mechanical Engineering, 41(4), 609-625.(2017)

[10]Kumar, S. R., Alexis, J. S., \&Thangarasu, V. S. Experimental Investigation of Influential Parameters in High Speed Machining of AMS 4205. Asian Journal of Research in Social Sciences and Humanities, 7(2), 508523. (2017)

[11]Ganeshkumar, S., Thirunavukkarasu, V., Sureshkumar, R., Venkatesh, S., \& Ramakrishnan, T. INVESTIGATION OF WEAR BEHAVIOUR OF SILICON CARBIDE TOOL INSERTS AND TITANIUM NITRIDE COATED TOOL INSERTS IN MACHINING OF EN8 STEEL.

[12]Kumar, S., Alexis, J., \&Thangarasu, V. S. Prediction of machining parameters for A91060 in end milling. Advances in Natural and Applied Sciences, 10(6 SE), 157-164.(2016)

[13]Kumar, R. S., Thangarasu, V. S., \& Alexis, S. J. Adaptive control systems in CNC machining processes-a review. Advances in Natural and Applied Sciences, 10(6 SE), 120-130. (2016)

[14]Kumar, S., Alexis, J., \&DhanabalakrishnanK.P. APPLICATION OF GA \& ANN FOR THE
OPTIMIZATION OF CUTTING PARAMETERS FOR END MILLING OPERATION- A COMPARISON. International Journal of Applied Engineering Research, 10(20), 18092-18107.(2015)

[15]Hassan Ali, Floren Plaza, Anthony Mann. "Flow visualization and modelling of scrubbing liquid flow patterns inside a centrifugal wet scrubber for improved design" Chemical Engineering Science 173(2017) 98109.(2017)

16)ManishaBal, BhimCharanMeikap. "Prediction of hydrodynamic characteristics of a venturi scrubber by using CFD simulation" South African journal of chemical engineering 24(2017) 222-231.(2017)

[17]AvinashMoharana, ParidhiGoel, ArunK.Nayak. "Performance estimation of a venturi scrubber and its application to self-priming operation in decontaminating aerosol particulates"Nuclear Engineering and Design 320(2017) 165-182.(2017)

[18]SiriwatUnyaphan,ThanyawanTarnpradab, Fumitake Takahashi, Kunio Yoshikawa. "An investigation of low cost and effective tar removal techniques by venturi scrubber producing syngas microbubbles and absorbent regeneration for biomass gasification"Science Direct, Energy procedia 105(2017) 406-412.(2017)

[19]Zhenhui Luan, Xiao Liu, MengZhengLibo Zhu. "Numerical Simulation of Square Section Venturi Scrubber with Horizontal Spray"

Elsevier, Science Direct, procedia computer science 107(2017) 117-121.(2017)

[20]Futang XING, Yuheng LI, Dan MEI, Shunfeg GUI, Liya WANG. "Optimized Design for Heavy Mound Venturi" Thermal Science, Vol. 21, No. 4, PP. 18731878.(2017)

[21]Yanmin Zhou, Zhongning Sun, Haifeng GU, Zhuang Miao. "Performance of iodide vapour absorption in the venturi scrubber working in selfpriming mode" Annals of Nuclear Energy 87(2016) 426-434.(2016)

[22]Ramakrishnan, T., \&PavayeeSubramani, S.. Investigation of Physico-Mechanical and Moisture Absorption Characteristics of Raw and Alkali Treated New Agave AngustifoliaMarginata (AAM) Fiber. Materials Science, 24(1), 53-58. [SCI \& Scopus IF: 0.593].(2018)

[23]Ramakrishnan, T., \& Sampath, P. S. Dry Sliding Wear Characteristics of New Short Agave AngustifoliaMarginata (AAM) Fiber-Reinforced Polymer Matrix Composite Material. Journal of Biobased Materials and Bioenergy, 11(5), 391-399. [Scopus \& SCI, IF: 2.993](2017)

[24]Jeyakumar, R., Sampath, P. S., Ramamoorthi, R., \& Ramakrishnan, T. Structural, morphological and mechanical behaviour of glass fibre reinforced epoxy nanoclay composites. The International Journal of Advanced Manufacturing Technology, 93(1-4), 527535. [Scopus \& SCI, IF: 2.663](2017) 


\section{International Journal of Engineering Applied Sciences and Technology, 2020 \\ Vol. 4, Issue 11, ISSN No. 2455-2143, Pages 167-178 \\ Published Online March 2020 in IJEAST (http://www.ijeast.com)}

[25]Ramakrishnan, T., \& Sampath, P. S. Experimental investigation of mechanical properties of untreated new Agave AngustifoliaMarginatafiber reinforced epoxy polymer matrix composite material. Journal of Advances in Chemistry, 13(4), 6120-6126. [Scopus \& IF: 1.131] (2017)

[26]Ramamoorthi, R., Jeyakumar.R, \&Ramakrishnan,T. Effect of Nanoparticles on the Improvement of Mechanical Properties of Epoxy Based Fiber Reinforced Composites - A Review. International Journal for Science and Advance Research in Technology, 3(11), 1251- 1256. (2017)

[27]Ramakrishnan, T., Sampath, P. S., \&Ramamoorthi, R. Investigation of Mechanical Properties and Morphological Study of the Alkali Treated Agave AngustifoliaMarginataFiber Reinforced Epoxy Polymer Composites. Asian Journal of Research in Social Sciences and Humanities, 6(9), 461-472.(2016)

[28]N.P.Gulhane, H.S.Kadam, S.S.Kale. "Analysis of Pressure and Velocity at the Throat of Self-Priming Venturi Scrubber" Journal of Material Science and Mechanical Engineering, Volume 2, Number 6, PP 5761.(2015)

[29]Jihe Chen, Bin Yang, Zhong-an Jiang, Yapeng Wang. "Effect of External Cyclone Diameter on Performance of a Two-Stage Cyclone Separator"

ACS Omega, DOI: 10.1021/acsomega.9b02216.(2019)

[30]M.N.Nwigbo. "Design, Fabrication and Performance Evaluation of a Cyclone System for Saw Dust Emission Control" International Journal of Engineering and Modern Technology, Vol. 5, No. 1.(2019)

[31]H.Saputro, T.Firdani, R.Muslim, Y.Estriyanto, D.S.Wijayanto, S.Lasminimi, Khaniffudin. "The CFD Simulation of Cyclone Separator without and with the Counter-cone in the Gasification Process" Material Science and Engineering 288(2018) 012-142.(2018)

[32]Rahul Panchal, Zheng (Jeremy) Li . "Design and Development of Tangential Cyclone Dust Collector" International Journal of Engineering Research and Development, Vol. 14, Issue 9, PP. 53-57.(2018)

[33]Ramakrishnan, T \&Sampath, P.S. Thermogravimetric Analysis (TGA) and the Effect of Moisture Absorption on the Mechanical Properties of New Agave AngustifoliaMarginata 3 Fiber (AAMF) Reinforced Epoxy Polymer Composite Material, International Journal of Printing, Packaging \& Allied Sciences, 4(5), 3245-3256. [Global IF: 0.12] (2016)

[34]Ramakrishnan, T., Sathish, K., Sampath, P. S., \&Anandkumar, S. Experimental investigation and optimization of surface roughness of AISI 52100 alloy steel material by using Taguchi method. Advances in Natural and Applied Sciences, 10(6 SE), 130-138. (2016)

[35]Sathish, K., Ramakrishnan, T., \&Sathishkumar, S. Optimization of turning parameters to improve surface finish of $16 \mathrm{Mn} \mathrm{Cr} 5$ material. Advances in Natural and Applied Sciences, 10(6 SE), 151-157. (2016)
[36]S.Karthik Raja S.Balasubramani, S.Venkatesh, T.Ramakrishnan. Effect Of Cryogenic Tempering On Steel,International Journal of Mechanical and Civil Engineering, 2 (6), 98-113.(2015)

[37]ForamMaheshwari, AnkitaParmar . "A Review Study on Gas-Solid Cyclone Separator using Lapple Model" Journal for Review, Volume 04, Issue 04, ISSN: 2395-7549.(2018)

[38]Ismail khan, Chethan $\mathrm{K}$ M, Avinash $\mathrm{H}$ S, RayidMuneer. "Design and Fabrication of Improved Cyclone Separator to Control the Dust in Rice Mills"

International Journal of Innovative Research in Science, Engineering and Technology, Vol. 6, Issue 9, DOI: 10.15680/IJIRSET.2017.0609179.(2017)

[39]AbhijeetGayakwad, Dr.Shivarudraiah. "CFD Analysis of Symmetrical Tangential Inlet Cyclone Separator" International Research Journal of Engineering and Technology, Volume: 04, Issue: 08.(2017)

[40]Prachik.Ithape, S.B.Barve, S.S.Pande, A.R.Nadgire. "Effect of Geometric Parameters on the Performance of Cyclone Separator using CFD" International Journal of Current Engineering and Technology, E-ISSN 22774106, P-ISSN 2347-5161.(2017)

[41]Venkatesh, S., \&Sakthivel, M. 'Numerical Investigation and Optimization for Performance Analysis in Venturi Inlet Cyclone Separator', Desalination and Water treatment, Vol. 90, No. 9, pp. 168-179. [Desalination publication, Impact Factor: 1.631, Scopus]. (2017)

[42]Venkatesh, S., Sakthivel, M., Sudhagar, \&Ajith Arul Daniel,. 'Modification of the cyclone separator geometry for improving the performance using Taguchi and CFD approach', Particulate Science and Technology, Doi:10.1080/02726351.2018.1458354. [Taylor \& Francis publication, Impact Factor: 0.785, Scopus]. (2018)

[43]Venkatesh, S., Bruno Clement, I., Avinasilingam, M., \&Arulkumar, E. "Design of Experiment Technique for Improving the Performance of Stirling Engine", International Research Journal of Engineering and Technology,Vol. 4, No. 5, pp. 62-65. (2017)

[44]Venkatesh, S., Balasubramani, S., Venkatramanan, S., \&Gokulraj, L. "Standardization of hpx spool for lead time reduction of string test", Journal of Mechanical and Civil Engineering,Vol. 2, No. 6, pp. 6279.

[45]Kousalya Devi, S., Venkatesh, S., \&Chandrasekaran. P. "Performance Improvement of Venturi Wet Scrubber,'Journal of Mechanical and Civil Engineering, Vol. 2, No. 4, pp. 1-9. (2015)

[46]Arunkumar, P., Dhachinamoorthi, P., Saravanakumar, K., \&Venkatesh, S.“Analysis and Investigation of Centrifugal Pump Impellers Using CFD," Engineering Science and Technology: An International Journal, Vol. 4, No. 4, pp. 112-117. (2014) [47]Dhanabalakrishnan,K.P., Abuthakir, J., Subramanian, R., Venkatesh, S."Evaluation of Tensile Properties of Particulate Reinforced Al-Metal Matrix 


\section{International Journal of Engineering Applied Sciences and Technology, 2020 \\ Vol. 4, Issue 11, ISSN No. 2455-2143, Pages 167-178 \\ Published Online March 2020 in IJEAST (http://www.ijeast.com)}

Composites," Engineering Science and Technology: An International Journal, Vol. 5, No. 1, pp. 173-175.(2015) [48]OsezuaO.Ibhadode, Emmanuel O.B.Ogedengbe, Marc A.Rosen. "Performance Characterization of GasSolid Cyclone for Separation of Particle from Syngas produced from Food Waste Gasifier Plant" European Journal of Sustainable Development, DOI: 10.20897/ejosdr.201713.(2017)

[49]M.Tahir, Mursalim, Salengake, Metusalach. "Design And Performance Of A Cyclone Separator Integrated With Heat Exchanger For Smoked Fish Production" ARPN Journal of Engineering and Applied Sciences. Vol. 12, No. 19, ISSN 1819-6608.(2017) [50]F.JustinDhiraviam, V.Naveenprabhu, M.Santhosh," Study the Effects of Solar Assisted Vapour Compression Air Conditioning System for Winter Applications", International Journal for Scientific Research \& Development|, Vol 4(11),(2017), pp. 505508

[51]V.NaveenPrabhu, K. SaravanaKumar, T. Suresh and M. Suresh," Experimental investigation on tube-intube heat exchanger using nanofluids", Advances in Natural and Applied Sciences, , Vol 10(7),(2016), pp. 272-278

[52]V.Naveenprabhu, D Mugeshkumaar, KB Pravin, V Ranjith, S Sanjay ArthanariSwamy," A Review of Evaporative Cooling of Finned and Non-Finned Heat Exchanger on Condenser", International Journal for Scientific Research \& Development, Vol 6(2),(2018), pp. 459-461.

[53]V.Naveenprabhu ,F.JustinDhiraviam, A.Vimal, K.Kumarrathinam," Design Of Common Header Line For Reduction Of Process Time In Pump Testing", International Research Journal of Engineering and Technology, Vol 4(1),(2017), pp. 969-975.

[54] Lin Liu, Hua-Shu Dou, Xiaoping chen. "Effect of particle diameter and injection position on the separation performance of cyclone separators" The Journal of Computational Multiphase Flows, Vol. 8(1) 40-47, DOI: 10.1177/1757482X16634199.(2016)

[55]G. B.Sakura, Andrew Y.T.Leung. "Experimental Study of Particle Collection Efficiency of Cylindrical Inlet Type Cyclone Separator" International Journal of Environmental science and Development. Vol. 06, No. 3, DOI: 10.7763/IJESD.2015.V6.581.(2015)

[56]B.Santhosh Kumar, et.al," Effect of Load on Joint Efficiency and Hardness in Friction Stir Welding of AA6061 \& AA6063 Aluminium Alloys.", International Journal for Scientific Research \& Development|, Vol 6(2),(2018), pp. 2669-2771.

[57]Ganesh Kumar, S \&Thirunavukkarasu, V, Investigation of Tool Wear and Optimization of Process Parameters in Turning of EN8 and EN 36 Steels Asian Journal of Research In Social Sciences And Humanities‘. vol. 6, no.11, pp. 237 - 243, Impact Factor: 0.315. (2016)

[58]Kumar, S. D., Kumar, S. S., \& Kumar, K. A. Investigation of Forced Frequency in a Commercial
Vehicle Suspension System. Mechanics and Mechanical Engineering, 22(4), 967974.(2018)

[59]Balasubramani, S., \&Balaji, N. Investigations of vision inspection method for surface defects in image processing techniques-a review. Advances in Natural and Applied Sciences, 10(6 SE), 115-120.(2016)

[60]Haibo Wu, Yi Liu, Wei Chen, Hanxiao Liu, ZhaohuiLui. "Experimental Study on Dust Removal of Flue Gas under O2/CO2 Combustion" Energy and Fuels, DOI: 10.1021/acs.energyfuels.9b02767.(2019)

[61]Balasubramani, S., Dhanabalakrishnan K.P., Balaji,N. Optimization of Machining parameters in Aluminium HMMC using Response Surface Methodology. International journal of applied engineering research, 10(20), 19736-19739. (2015)

[62]Subramaniam, B., Natarajan, B., Kaliyaperumal, B., \&Chelladurai, S. J. S. Investigation on mechanical properties of aluminium 7075-boron carbide-coconut shell fly ash reinforced hybrid metal matrix composites. China Foundry, 15(6), 449-456. (2018)

[63]Sureshbabu, Y., \&AshokaVarthanan, P. Study the emission characteristics of catalytic coated piston and combustion chamber of a four stroke spark ignition (SI) engine. Journal of Chemical and Pharmaceutical Sciences ISSN, 974, 2115.

[64]SerhatGül, FehmiAkgün, Emir Aydar, Namikunlu. "Pressurized gasification of lignite in a pilot scale bubbling fluidized bed reactor with air, oxygen, steam and CO2 agents" Applied Thermal Engineering, DOI: https://doi.org/10.1016/j.applthermaleng.2017.11.021.(2 017)

[65]Sureshbabu, Y., \&AshokaVarthanan, P. Study the emission characteristics of catalytic coated piston and combustion chamber of a four stroke spark ignition (SI) engine. International Journal for Scientific Research \& Development, 6(02), 1981-1983.(2018) 